\title{
Oral airway resistance during wakefulness in patients with obstructive sleep apnoea
}

\author{
T C Amis, N O’Neill, J R Wheatley
}

\begin{abstract}
Background-Patients with obstructive sleep apnoea (OSA) have a number of upper airway structural abnormalities which may influence the resistance of the oral airway to airflow. There have been no systematic studies of the flow dynamics of the oral cavity in such patients.

Methods-Inspiratory oral airway resistance to airflow $\left(R_{o}\right)$ was measured in 13 awake patients with OSA in both the upright and supine positions (neck position constant). Each subject breathed via a mouthpiece while the nasal airway was occluded with a nasal mask.

Results-In the upright position the mean (SE) $R_{\mathrm{o}}$ was $1.26(0.19) \mathrm{cm} \mathrm{H}_{2} \mathrm{O} / \mathrm{l} / \mathrm{s}$ (at $0.4 \mathrm{1} / \mathrm{s})$ which increased to 2.01 $(0.43) \mathrm{cm} \mathrm{H}_{2} \mathrm{O} / 1 / \mathrm{s}$ when supine $(\mathrm{p}<0.05$, paired $t$ test). The magnitude of this change correlated negatively with the respiratory disturbance index $(r=-0.60, \mathrm{p}=$ 0.03).

Conclusion-In awake patients with OSA $R_{o}$ is normal when upright but abnormally raised when in the supine position.

(Thorax 1999;54:423-426)
\end{abstract}

Keywords: obstructive sleep apnoea; oral airway resistance; body position

The role of upstream resistance in the pathophysiology of inspiratory narrowing/collapse of the pharyngeal airway in patients with obstructive sleep apnoea (OSA) has been explored by a number of investigators, almost exclusively in terms of the influence of nasal airflow resistance on sleep disordered breathing events. ${ }^{1-3}$ During nasal breathing the nasal passages constitute the relevant upstream inspiratory resistor whereas during mouth breathing the oral cavity is the potential site of upstream resistance.

The resistance to airflow through the oral cavity $\left(R_{O}\right)$ is a major component of total upper airway resistance during oral and oronasal breathing. ${ }^{45}$ Mouth breathing occurs during sleep, even in normal subjects, and may be associated with an increased incidence of sleep disordered breathing events. ${ }^{6}$ Patients with OSA frequently have mandibular/orthodontic abnormalities $^{78}$ and enlargement of the tongue, ${ }^{8}$ all of which could contribute to an increased $R_{O}$. During periods of oral breathing while asleep a high $R_{0}$ may be associated with more negative inspiratory intraluminal pressures in the oropharynx and hypopharynx, thus increasing susceptibility to airway narrowing and collapse. Furthermore, if this increased $R_{O}$ was primarily associated with structural changes in the upper airway-for example, enlargement of the tongue - then $R_{\mathrm{O}}$ might also be increased during wakefulness in patients with OSA.

While total upper airway resistance, ${ }^{9}$ nasal resistance, ${ }^{3}$ and pharyngeal resistance ${ }^{10}$ have all been studied extensively in OSA, there have been no systematic studies of the flow dynamics of the oral cavity in such patients. In the present study we have measured $R_{O}$ in a group of patients with OSA during wakefulness, examined the influence of posture on $R_{O}$, and studied the fluid mechanics of oral airflow in OSA.

\section{Methods}

SUBJECTS

Inspiratory $R_{0}$ was measured in both the upright and supine positions in 13 awake patients (10 men) of mean (SE) age 51.0 (3.2) years, body mass index (BMI) $37.3(1.6) \mathrm{kg} /$ $\mathrm{m}^{2}$, with symptoms of moderate to severe OSA. The diagnosis was confirmed by overnight polysomnography ${ }^{11}$ and $R_{\mathrm{O}}$ measurements were performed within a four month period of the polysomnography. No patient was undergoing treatment with nasal continuous positive airway pressure (CPAP) at the time the measurements were made and none wore dental plates. Informed consent was obtained from each subject and the protocol was approved by the Western Sydney Area Health Service human ethics committee.

MEASUREMENT OF INSPIRATORY $\mathrm{R}_{\mathrm{O}}$

Each study was performed with the subject breathing quietly via a standard mouthpiece (Sensor Medics, internal cross sectional area $300 \mathrm{~mm}^{2}$, Middle Park, Victoria, Australia). The mouthpiece was connected to a heated pneumotachograph (Fleisch \#2, Gould, Bilthoven, the Netherlands) which was coupled to a differential pressure transducer $\left( \pm 10 \mathrm{~cm} \mathrm{H}_{2} \mathrm{O}\right.$, Celesco Transducer Products, IDM Instruments, Dandenong, Victoria, Australia) for the measurement of oral airflow. An occluded nasal CPAP mask (Sullivan, ResMed, Sydney, NSW, Australia) was placed over the nose and checked to ensure the absence of leaks. With the occluded nasal mask in place, only oral breathing was possible. Since there was no nasal route airflow, pressure measured inside the mask reflected oropharyngeal pressure. Transoral pressure was then measured using a differential pressure transducer (MP $45, \pm 100 \mathrm{~cm} \mathrm{H}_{2} \mathrm{O}$, Validyne, Northridge, California, USA), one side of which was connected to the mouthpiece while the other side was 
connected to the nasal mask. Both flow and pressure signals were digitised using a sampling frequency of $50-100 \mathrm{~Hz}$ and recorded directly on a computer. The data were stored on disk for subsequent analysis.

PROTOCOL

Subjects were studied first in the upright (seated) position and then supine. Neck position was maintained constant throughout the study by ensuring that the measured distance from the chin (tip of mandible) to the manubrio-sternal notch remained unchanged $(9-11 \mathrm{~cm})$.

\section{DATA ANALYSIS}

Inspiratory $R_{O}$ was calculated directly from pressure-flow plots reconstructed from the stored data. ${ }^{12}$ An inspiratory transoral pressure-flow plot was constructed from data obtained during 4-5 consecutive stable and representative breaths from each run. A power function of the form $\mathrm{P}=a \dot{\mathrm{V}}^{b}$ (where $\mathrm{P}$ is transoral pressure, $\dot{\mathrm{V}}$ is oral flow, and $a$ and $b$ are constants) was then fitted to the inspiratory transoral pressure-flow curve by the method of least squares. Only data exhibiting no phase lag between the pressure and flow signals (that is, no looping of the transoral pressure-flow plot around zero flow) were accepted for analysis. In this manner data which may have been influenced by partial narrowing of the nasopharyngeal airway were excluded. Inspiratory $R_{\mathrm{O}}$ was then calculated from this relationship at a flow rate of $0.41 / \mathrm{s}$. In all, 3-6 separate measurements were obtained in the upright position and 2-5 when supine. The results from repeated runs were then averaged to give individual mean values.

Statistical comparisons were made using the Student's $t$ test for paired samples. The relationship between the level of $R_{O}$ and respiratory disturbance index was examined using simple linear regression analysis. $p$ values of $<0.05$ were considered significant.

\section{Results}

The respiratory disturbance index (RDI; apnoeas plus hypopnoeas) was 62.0 (7.5) events/ hour of sleep (range 14-103). It was similar during non-REM sleep (61.2 (8.3) events/ hour) and REM sleep (63.2 (7.5) events/hour) and was no different during supine sleep (70.6 (7.1) events/hour) and non-supine sleep (53.3 (10.9) events/hour; both $\mathrm{p}>0.05)$. On average, patients spent 70.4 (6.2)\% of total sleep time in the supine position (range $30-100 \%$ of total sleep time). There was a significant negative correlation between the total RDI and the percentage of total sleep time in the supine position $(\mathrm{p}<0.007)$.

In the upright (seated) position inspiratory $\mathrm{R}_{\mathrm{O}} \quad$ (at $0.4 \mathrm{1} / \mathrm{s}$ ) ranged from 0.33 to $2.90 \mathrm{~cm} \mathrm{H}_{2} \mathrm{O} / 1 / \mathrm{s}$ and in the supine position from 0.59 to $4.55 \mathrm{~cm} \mathrm{H}_{2} \mathrm{O} / 1 / \mathrm{s}$. The within subject coefficient of variation $(\mathrm{CV})$ for $\mathrm{R}_{\mathrm{O}}$ was $26.7(3.0) \%$ in the upright position and 32.1 (8.2) $\%$ in the supine position. In moving from the upright to the supine position, $\mathrm{R}_{\mathrm{O}}$ increased (by $>0.1 \mathrm{~cm} \mathrm{H} \mathrm{H}_{2} \mathrm{O} / \mathrm{l} / \mathrm{s}$ ) in nine patients, de-

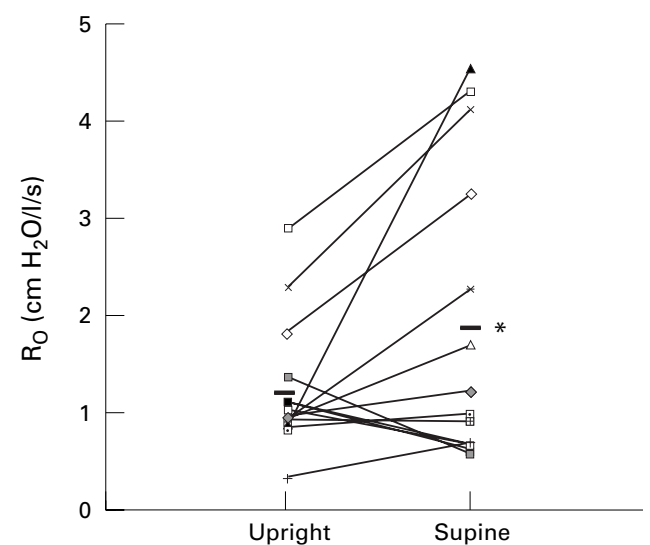

Figure 1 Oral resistance $\left(R_{0}\right)$ at $0.4 \mathrm{l} / \mathrm{s}$ in the upright and supine positions in 13 awake patients with obstructive sleep apnoea. Different symbols represent individual subjects. Horizontal bars denote mean values. In most of the patients $R_{O}$ increased from the upright to the supine posture but there is considerable variability in the magnitude of the change. ${ }^{\star} p<0.05$ compared with upright position.

creased (by $>0.1 \mathrm{~cm} \mathrm{H} \mathrm{H}_{2} \mathrm{O} / 1 / \mathrm{s}$ ) in three patients, and did not change in one patient (fig 1). For the whole group the mean $R_{O}$ was 1.26 (0.19) $\mathrm{cm} \mathrm{H}_{2} \mathrm{O} / 1 / \mathrm{s}$ (CV 55.4\%) in the upright position and this increased significantly to 2.01 (0.43) $\mathrm{cm} \mathrm{H}_{2} \mathrm{O} / 1 / \mathrm{s}(\mathrm{CV} 76.4 \%$; $<<0.05)$ in the supine position (fig 1 ).

The power function fitted the data with an $R^{2}$ value of $>0.94$ for the upright position and $>0.91$ for the supine position across all the runs. The values for the $a$ constant ranged from 1.43 to 4.49 when upright and from 1.16 to 11.43 when supine. For the whole group the mean a constant increased significantly from the upright $(2.21(0.26))$ to the supine (3.85 $(0.88))$ position $(\mathrm{p}<0.04)$. The values for the $b$ constant ranged from 1.38 to 2.62 in the upright position and from 1.36 to 2.38 in the supine position. There was no significant difference between the mean values for the $b$ constant when upright $(1.70(0.10))$ and supine (1.77 (0.07)).

There was no relationship between awake upright $\mathrm{R}_{\mathrm{O}}$ and RDI $(r=-0.09, \mathrm{p}>0.7)$. However, when supine there was a borderline significant trend $(r=-0.52, \mathrm{p}=0.07)$ for those individuals with a higher $\mathrm{R}_{\mathrm{O}}\left(>2.0 \mathrm{~cm} \mathrm{H}_{2} \mathrm{O} / \mathrm{l} / \mathrm{s}\right)$ to be the least severely affected by their disease (RDI $<57$ events/hour). This negative relationship between a high $R_{O}$ when supine and disease severity was stronger when the correlation between the absolute change in $R_{O}$ (in moving from upright to supine) and RDI was examined ( $r=-0.60, \mathrm{p}=0.03$, fig 2$)$. Thus, those individuals with no change or only a small increase or decrease in $R_{O}$ when moving to the supine position tended to have a higher RDI than did those patients in whom the $R_{O}$ increased substantially.

There was also a significant positive relationship between BMI and RDI $(r=0.62, \mathrm{p}=$ 0.03 ) and a significant negative relationship between $\mathrm{BMI}$ and $\mathrm{R}_{\mathrm{O}}$ when supine $(r=-0.56$, $\mathrm{p}=0.05)$, as well as the change in $\mathrm{R}_{\mathrm{o}}$ in moving from upright to supine $(r=-0.63, \mathrm{p}=$ 0.02). 


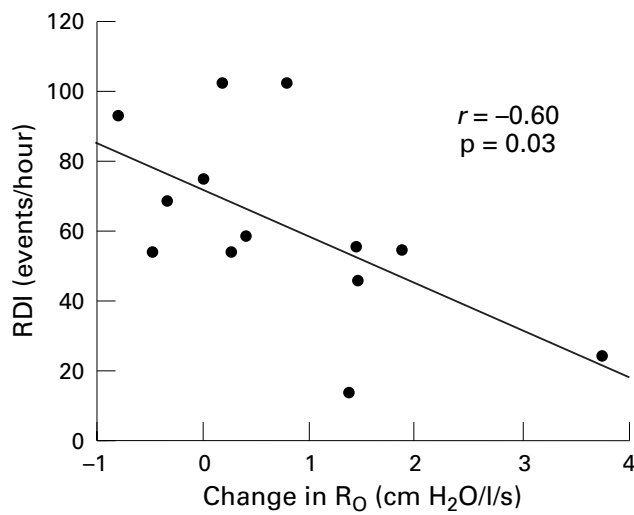

Figure 2 Change in oral resistance $\left(R_{\alpha}\right)$ at $0.4 \mathrm{l} / \mathrm{s}$ from upright to supine positions during wakefulness in 13 OSA patients plotted against respiratory disturbance index (RDI). Linear regression line, correlation coefficient ( $r$ ), and $p$ value are shown.

\section{Discussion}

Measurements of $R_{O}$ were obtained with patients awake and breathing on a standard mouthpiece. A mouthpiece was used in order to standardise the degree of mouth opening. This approach provides a measurement of $R_{O}$ which is reflective of structures posterior to the teeth. We hypothesised that, if $R_{O}$ is raised in awake subjects with OSA, it would be because of anatomical abnormalities posterior to the dental arcades - for example, tongue enlargement. Consequently, our study focuses on $R_{O}$ measurements obtained with the lips and teeth in a constant and standardised position and in awake subjects. It should therefore be emphasised that our findings may not reflect the situation during sleep.

The increase in $R_{O}$ found with change in body position in patients with OSA is in contrast to results obtained in normal subjects using the same technique. ${ }^{13}$ In this latter study there was no significant difference in $R_{O}$ when upright and supine in a group of 17 normal men. The mean value for upright $\mathrm{R}_{\mathrm{O}}$ measured in the present study $\left(1.26(0.19) \mathrm{cm} \mathrm{H}_{2} \mathrm{O} / \mathrm{l} / \mathrm{s}\right)$ was slightly higher than that obtained in the normal subjects $\left(0.86(0.23) \mathrm{cm} \mathrm{H}_{2} \mathrm{O} / \mathrm{l} / \mathrm{s}\right)$, perhaps because of slightly more head and neck flexion in the patients (chin to manubriosternal notch distance of $9-11 \mathrm{~cm}$ in patients compared with $14 \mathrm{~cm}$ in normal subjects). Alternatively, the trend for a higher $R_{O}$ in patients with OSA might reflect real anatomical differences between the patients and the normal subjects. The previously studied normal subjects were younger (36 (2) years) and had a smaller BMI $\left(26.4(0.9) \mathrm{kg} / \mathrm{m}^{2}\right)$ than the patients in the present study. It is therefore possible that the difference between the two studies is a reflection of anthropometric characteristics rather than OSA per se. In any case, the mean supine value (2.01 $\left.(0.43) \mathrm{cm} \mathrm{H}_{2} \mathrm{O} / \mathrm{l} / \mathrm{s}\right)$ in the patients was double that measured in the normal subjects $(0.90$ $\left.(0.16) \mathrm{cm} \mathrm{H}_{2} \mathrm{O} / \mathrm{l} / \mathrm{s}\right) .{ }^{13}$ Thus, when awake and upright, patients with OSA and a high BMI have a relatively normal $R_{0}$. However, unlike normal subjects, on assuming the supine position the $\mathrm{R}_{\mathrm{O}}$ increases.
It has long been recognised that patients with OSA tend to have a reduced upper airway cross sectional area ${ }^{14-17}$ compared with matched control subjects, even while awake and upright. When the anatomy of the upper airway of patients with OSA is compared with that of normal subjects, most attention has been focused on the retropalatal and retroglossal airway segments since these regions are the principal sites of occlusion during obstructive apnoeas. ${ }^{10}$ In general, patients with OSA have smaller pharyngeal airways which are more collapsible, ${ }^{18}{ }^{19}$ are shaped differently, ${ }^{20}$ and are more likely to be narrowed in the supine position $^{21-23}$ than those of normal subjects.

The anatomical abnormalities of the upper airway in patients with OSA are associated with an increased upper airway resistance to airflow. ${ }^{9}$ While normal subjects maintain a constant upper airway resistance between the upright and supine positions, pharyngeal resistance tends to increase in patients with OSA when they are supine. ${ }^{92}$ The present study demonstrates that oral airway resistance behaves in a similar manner provided the head, neck, lip, and jaw position is maintained constant. This is in agreement with a brief report by Kawano et $a l^{25}$ who also found an increase in $R_{\mathrm{O}}$ (measurement method not described) when patients with OSA moved from the upright to the supine position.

A feature of the difference in upper airway anatomy between patients with OSA and normal subjects is tongue size, patients with OSA having a greater tongue cross sectional area $^{26}$ which may be related to airway inflammation and/or oedema ${ }^{27}$ or an adaptive increase in muscle mass related to upper airway muscle hyperactivity. ${ }^{28}$ In addition, Pae et $a l^{23}$ have shown that the cross sectional area of the tongue of patients with OSA increased by $4.3 \%$ while the oropharyngeal area decreased by $36.5 \%$ when changing from the upright to the supine position, but no changes were observed in normal subjects. These findings suggest that changes in tongue size or position may be responsible for the increase in $R_{O}$ found in the patients in the present study when in the supine position.

A feature of our findings was the negative relationship between the change in $R_{O}$ when in the supine position and the severity of OSA as measured by RDI. A potential explanation for this finding may lie in the response of the tongue to changes in posture. Tongue position depends on the degree of recruitment of genioglossus muscle activity. Assumption of the supine position has been shown to recruit genioglossus muscle activity in both normal subjects and patients with OSA. ${ }^{29}$ This response is thought to help preserve oropharyngeal dimensions. Indeed, oropharyngeal diameter has been shown to increase in normal subjects and patients with OSA $^{21}$ in the supine position. However, in other studies the oropharynx has been found to narrow in some OSA patients in the supine position. ${ }^{23}$ Thus, there appears to be a heterogeneous response by patients with OSA to the supine position, the oropharynx widening in some individuals 
and narrowing in others. In their study of genioglossus muscle recruitment Douglas et a ${ }^{29}$ found that, while most patients with OSA had substantially increased genioglossus electromyographic activity in the supine position, some patients did not. We speculate that patients who maintain their pharyngeal dimensions in the supine position do so by recruiting genioglossus muscle activity which moves the base of the tongue forward into the oral cavity and away from the posterior pharyngeal wall. Indeed, voluntary tongue protrusion does lead to an increase in cross sectional area of the oropharynx in awake patients with OSA when supine. ${ }^{30}$ When lip and teeth position are fixed, this movement of the tongue may result in a narrowing of the oral cavity (although a widening of the oropharynx) and an increase in $R_{0}$ (although a decrease in pharyngeal resistance), especially in individuals with a large tongue.

Since during sleep airflow is predominantly via the nasal pathway and occlusive apnoeas are predominantly related to pharyngeal collapse, patients in whom the $R_{0}$ increases in the supine position may protect their pharyngeal airway from collapse more effectively than subjects who are unable to mount such a response and therefore preserve $R_{0}$, but with narrowing of the pharyngeal airway. These proposed mechanisms, however, need to be validated with direct experimental testing. In addition, it is not clear if the response is related to anthropometric characteristics (since there was also a significant negative relationship between BMI and supine $R_{0}$ ) or to the severity of OSA per se.

In contrast to our previous study in normal subjects, ${ }^{12}$ values for the $a$ constant of the fitted power function in the present study also increased significantly when patients moved from the upright to the supine position. This finding confirms that $R_{O}$ increases in the supine position at all the flow rates encountered. ${ }^{12}{ }^{31}$ The values for the $b$ constant were in the range indicating a turbulent to orifice flow regime ${ }^{31}$ and were unaffected by body position. This contrasts with our previous study of normal subjects ${ }^{12}$ in which $b$ values did increase in the supine position. Thus, during mouthpiece breathing a turbulent flow regime exists in the oral cavity in patients with OSA, as it does in normal subjects. ${ }^{12}$

This study was supported by the National Health and Medical Research Council of Australia, the Community Health \& AntiTuberculosis Association of New South Wales, and the Garnet Passe \& Rodney Williams Foundation. The authors wish to thank Emily Di Somma for assistance with preparation of the manuscript.

1 Zwillich CW, Pickett C, Hanson FN, et al. Disturbed sleep and prolonged apnea during nasal obstruction in normal men. Am Rev Respir Dis 1981;124:158-60.

$2 \mathrm{McNicholas}$ WT, Tarlo S, Cole P, et al. Obstructive apnoeas during sleep in patients with seasonal allergic rhinitis. Am Rev Respir Dis 1982;126:624-8.

3 Atkin M, Taskar V, Clayton N, et al. Nasal resistance in obstructive sleep apnoea. Chest 1994;105:1133-5.
4 Schiratzki $\mathrm{H}$. The oral and laryngeal components of the pper airway resistance during mouth breathing. Acta upper airway resistance duri

5 Spann R, Hyatt R. Factors affecting upper airway resistance in conscious man. F Appl Physiol 1971;31:708-12.

6 Gleeson K, Zwillich CW, Braier K, et al. Breathing route during sleep. Am Rev Respir Dis 1986;134:115-20.

7 Riley R, Guilleminault C, Herran J, et al. Cephalometric analysis and flow volume loops in obstructive sleep apnea patients. Sleep 1983;6:303-11.

8 Lowe AA, Fleetham JA, Adachi S, et al. Cephalometric and computed tomographic predictors of obstructive sleep apnea severity. Am 7 Orthod Dentofac Orthop 1995;107: apnea seve.

9 Anch AM, Remmers JE, Bunce III H. Supraglottic airway resistance in normal subjects and patients with occlusive sleep apnea. F Appl Physiol 1982;53:1158-63.

10 Hudgel DW. Variable site of airway narrowing among obstructive sleep apnea patients. F Appl Physiol 1986;61: 1403-9.

11 Phillipson EA, Remmers JE. American Thoracic Society Position Paper: indications and standards for cardiopulmonary sleep studies. Am Rev Respir Dis 1989;139:559-68.

12 Wheatley JR, Amis TC, Engel LA. Nasal and oral airway pressure-flow relationships. F Appl Physiol 1991;71:231724

13 Amis TC, O'Neill N, Wheatley JR. Oral airway flow dynamics in normal humans. F Physiol 1999;515:293-8.

14 Haponik EF, Smith PL, Bohlman ME, et al. Computerized tomography in obstructive sleep apnea: correlation of airway size with physiology during sleep and wakefulness. Am Rev Respir Dis 1983;127:221-6.

15 Suratt PM, Dee P, Atkinson RL, et al. Fluoroscopic and computed tomographic features of the pharyngeal airway in obstructive sleep apnea. Am Rev Respir Dis 1983;127: 487-92.

16 Hoffstein V, Zamel N, Phillipson EA. Lung volume dependence of pharyngeal cross-sectional area in patients with obstructive sleep apnea. Am Rev Respir Dis 1984;130:1758.

17 Martin SE, Marshall I, Douglas NJ. The effect of posture on airway calibre with the sleep apnea/hypopnea syndrome. Am 7 Respir Crit Care Med 1995;152:721-4.

18 Suratt PM, McTier RF, Wilhoit SC. Collapsibility of the nasopharyngeal airway in obstructive sleep apnea. Am Rev Respir Dis 1985;132:967-71.

19 Bradley TD, Brown IG, Zamel N, et al. Differences in pharyngeal properties between snorers with predominantly central sleep apnea and those without sleep apnea. Am Rev Respir Dis 1987;135:387-91.

20 Pevernagie DA, Stanson AW, Seedy II PF, et al. Effects of body position on the upper airway of patients with obstructive sleep apnea. Am f Respir Crit Care Med 1995;152:17985.

21 Yildirim N, Fitzpatrick MF, White KF, et al. The effect of posture on upper airway dimensions in normal subjects and
in patients with the sleep apnea/hypopnea syndrome. $\mathrm{Am}$ in patients with the sleep apne
Rev Respir Dis 1991;144:845-7.

22 Miyamoto K, Ozbek MM, Lowe AA, et al. Effect of body position on tongue posture in awake patients with obstructive sleep apnoea. Thorax 1997;52:255-9.

23 Pae E, Lowe AA, Sasaki K, et al. A cephalometric and electromyographic study of upper airway structures in the upright and supine positions. Am $\mathcal{F}$ Orthod Dentofac Orthop 1994;106:52-9.

24 Leiter JC, Knuth SL, Bartlett Jr D. Dependence of pharyngeal resistance on genioglossal EMG activity, nasal geal resistance on genioglossal EMG activity, na
resistance, and airflow. $\mathcal{f}$ Appl Physiol 1992;73:584-90.

25 Kawano K, Usui N, Kanazawa $\mathrm{H}$, et al. Changes in nasal and oral respiratory resistance before and after uvulopalatopharyngoplasty. Acta Otolaryngol (Stockh) Suppl 1996;523: $236-8$.

26 De Berry-Borowiecki B, Kukwa A, Blanks RHI. Cephalometric analysis for diagnosis and treatment of obstructive sleep apnea. Laryngoscope 1988;98:226-34.

27 Ryan CF, Lowe AA, Li D, Fleetham JA. Magnetic resonance imaging of the upper airway in obstructive sleep apnea before and after chronic nasal continuous positive airway pressure therapy. Am Rev Respir Dis 1991;144:939-44.

28 Petrof BJ, Hendricks JC, Pack AI. Does upper airway muscle injury trigger a vicious cycle in obstructive sleep apnea? A hypothesis. Sleep 1996;19:465-71.

29 Douglas NJ, Jan MA, Yildirim N, et al. Effect of posture and breathing route on genioglossal electromyogram activity in normal subjects and in patients with the sleep apnea/ hypopnea syndrome. Am Rev Respir Dis 1993;148:1341-5.

30 Ferguson KA, Love LL, Ryan CF. Effect of mandibular and tongue protrusion on upper airway size during wakefulness. Am $\mathcal{F}$ Respir Crit Care Med 1997;155:1748-54.

31 Jaeger MC, Matthys $H$. The pattern of flow in the upper human airways. Respir Physiol 1968/1969;6:113-27. 